\title{
What will it take? Using an implementation research framework to identify facilitators and barriers in implementing a school- based referral system for sexual health services
}

Emily Leung ${ }^{1 *}$, Kathryn J. Wanner ${ }^{2}$, Lindsay Senter ${ }^{1}$, Amanda Brown ${ }^{1}$ and Dawn Middleton ${ }^{1}$

\begin{abstract}
Background: Across the United States, sexually transmitted infections and unintended pregnancy rates are alarmingly high among youth. Schools, due to their proximity and access to youth, can increase student access to sexual health services (SHS) by creating referral systems (RS) to link students to school- and community-based SHS. From 2013 to 2018, the Centers for Disease Control and Prevention's Division of Adolescent and School Health funded 17 Local Education Agencies (LEA) to partner with priority schools and stakeholders to develop and implement RS to increase student access to SHS. Cicatelli Associates Inc. (CAI) was funded to provide capacity-building to LEA. In 2016-2017, CAI conducted case studies at two LEA, both large and urban sites, but representing different geographical and political contexts, to elucidate factors that influence RS implementation.
\end{abstract}

Methods: Nineteen LEA and community-based healthcare (CBH) staff were interviewed in the Southeastern $(n=9)$ and Western U.S. $(n=10)$. Key constructs (e.g., leadership engagement, resources, state and district policies) across the five domains of the Consolidated Framework for Implementation Research (CFIR) framework guided the methodology and analysis. Qualitative data was analyzed using the Framework Method and contextual factors and themes that led to RS implementation were identified.

Results: Interviewees strongly believed that school-based RS can decrease STI, HIV and unintended pregnancy and increase students' educational attainment. We identified the following contextual key factors that facilitate successful implementation and integration of an RS: enforcing state and district policies, strong LEA and CBH collaboration, positive school culture towards adolescent health, knowledgeable and supportive staff, leveraging of existing resources and staffing structures, and influential district and school building-level leadership and champions. Notably, this case study challenged our initial assumptions that RS are easily implemented in states with comprehensive SHS policies. Rather, our conversations revealed how districts and local-level policies can have significant impact and influence to impede or promote those policies.

(Continued on next page)

\footnotetext{
* Correspondence: ELeung@caiglobal.org

'Cicatelli Associates, Inc. (CAl), 505 Eighth Ave. Suite 1900, New York, NY

10018, USA

Full list of author information is available at the end of the article
}

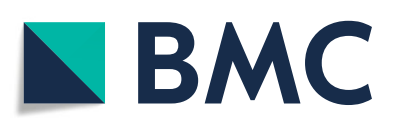

(- The Author(s). 2020 Open Access This article is licensed under a Creative Commons Attribution 4.0 International License, which permits use, sharing, adaptation, distribution and reproduction in any medium or format, as long as you give appropriate credit to the original author(s) and the source, provide a link to the Creative Commons licence, and indicate if changes were made. The images or other third party material in this article are included in the article's Creative Commons licence, unless indicated otherwise in a credit line to the material. If material is not included in the article's Creative Commons licence and your intended use is not permitted by statutory regulation or exceeds the permitted use, you will need to obtain permission directly from the copyright holder. To view a copy of this licence, visit http://creativecommons.org/licenses/by/4.0/ The Creative Commons Public Domain Dedication waiver (http://creativecommons.org/publicdomain/zero/1.0/) applies to the data made available in this article, unless otherwise stated in a credit line to the data. 
(Continued from previous page)

Conclusions: Through the use of the CFIR framework, the interviews identified important contextual factors and themes associated with LEAs' implementation barriers and facilitators. The study's results present key recommendations that other LEA can consider to optimize integration of RS-related evidence-based practices, systems, and policies in their districts.

Keywords: Consolidated framework for implementation science, School health, Implementation readiness, Implementation tools, Case study methods, Implementation science, School-based referral system

\section{Background}

Across the United States (U.S.), sexually transmitted infection (STI) and unintended pregnancy rates are alarmingly high for our nation's youth; young people aged 15-24 acquire half of all new STIs [1]. Among this population from 2013 to 2017, syphilis cases have nearly doubled, gonorrhea cases have increased by $67 \%$, and chlamydia remains the most common STI, with $45 \%$ diagnosed cases occurring among 15-24year old females [2]. Untreated, the sequalae of STIs include pelvic inflammatory disease, infertility, and cervical cancer [3, 4]. Additionally, among adolescents age 15-19, the unintended pregnancy rate is 41 per 1000 females, which is the second highest rate across all age groups [5]. Since almost $40 \%$ of high school students reported having engaged in sexual activity, the need for consistent and comprehensive STI and unintended pregnancy prevention services is high [6].

Schools play a critical role in improving access to sexual health services due to their proximity and access to youth. In the U.S., schools have direct contact with more than 15 million students attending grades 9-12 for at least $6 \mathrm{~h}$ a day during the key years of their social, physical, and intellectual development [7]. As identified by the U.S. Department of Health, improving access to sexual health services within school settings is "crucial" to eliminating disparities in reproductive health outcomes [8]. In recent years, school-based sexual health services, such as STI screenings, and School Based Health Centers (SBHC) that offer sexual and reproductive health services, have shown promise in increasing positive adolescent sexual health outcomes, including healthy sexual behaviors, contraceptive use, and decrease in unintended pregnancies [9-11]. However, there are myriad barriers and challenges to comprehensive access. While the number of SBHC is increasing, many schools, especially in rural areas, do not have SBHC, and many SBHC have limited or no provision of sexual health services [12]. Only $37 \%$ of SBHC are capable of distributing contraceptives onsite; almost $50 \%$ are prohibited from doing so by state or local policy, and even among schools that provide sexual health services, utilization of the services may be low [13].

One way for schools to overcome such barriers and to increase student access is by creating comprehensive referral systems to link students to sexual health servicesboth school- and community-based [14]. Recent research has shown promising findings for improving school-based referral systems; in one study, high school females who received referrals to sexual health services from school staff were more likely to receive birth control, get tested and/or treatment for STIs, and receive an HIV test [15]. Rasberry et al. [16] also found that students who received a referral from school staff were three times more likely to get tested for HIV and STIs, after controlling for factors such as sex, age, race/ethnicity, sexual minority status, and having had sex.

In a direct response to these trends, from 2013 to 2018, the Centers for Disease Control and Prevention's (CDC) Division of Adolescent and School Health funded the Promoting Adolescent Health through School-Based HIV/STI Prevention and School-Based Surveillance project. Through a competitive application process in response to CDC's notice of funding opportunity, 17 Local Education Agencies (LEA) received funding to partner with priority schools and other stakeholders to develop and implement a referral system and associated protocols, resources, and tools to increase student access to sexual health services. Cicatelli Associates, Inc. (CAI), was funded as the Technical Assistance Center to provide tools and capacity building to the LEAs.

In conjunction with the CDC, CAI developed the Core Components of a Referral System framework that outlines and describes the eight core components necessary for successful referral system implementation (see Fig. 1) [14]. The eight core components include: policy, referral staff, procedures, referral guide, communications and marketing, monitoring and evaluation, management and oversight, and organizational partnerships. Policies set forth expectations related to when and under what circumstances students can receive information from school staff about sexual health services, access schoolbased healthcare, and leave school premises to receive community-based medical services. Referral staff recognize adolescents in need of sexual health services and provide referrals. Procedures provide a standardized and concise process for school staff to follow to connect students to appropriate sexual health services. A referral guide serves as an important tool for staff to select appropriate service providers for students. A communications and marketing strategy can help to raise awareness of availability of both school- and community-based 


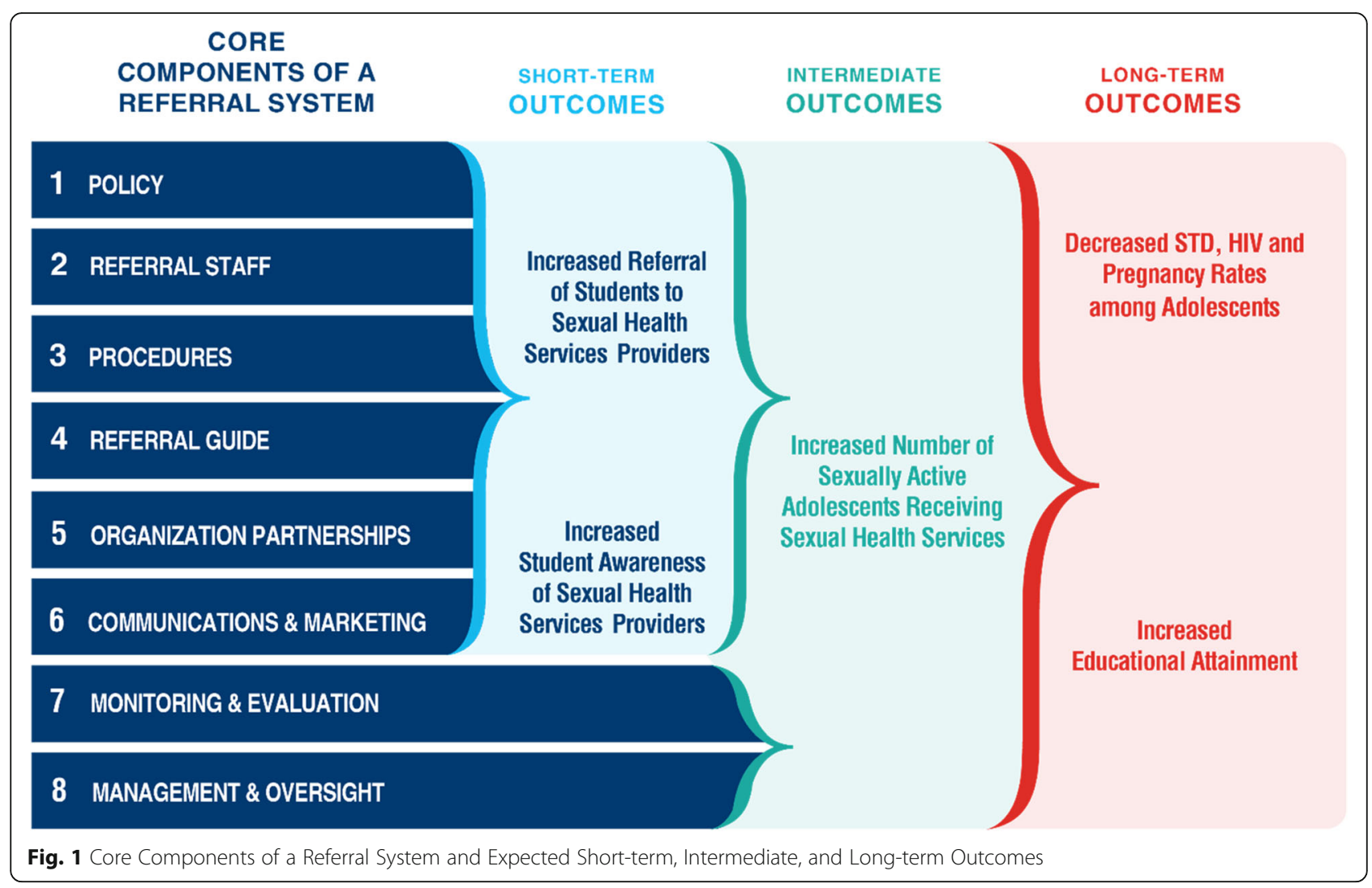

adolescent-friendly sexual health services. Establishing a monitoring and evaluation plan and system allows for evaluating the extent to which the referral system is achieving its intended objectives. Management and oversight ensure that the referral system is being implemented in a standardized manner and is on track to achieve outcomes. Lastly, strategic organizational partnerships between schools, school districts, SBHC, health departments, and youth-friendly communitybased health providers (CBHP) are needed so that students can access needed services at schools or in the community.

This framework is intended to provide LEA with a standardized approach for implementing a referral system, with the short-term goals of increasing student awareness of school- and community-based sexual health service providers and increasing student referrals to school and community-based sexual health service providers, and the intermediate-term goal of increasing the number of sexually active adolescents receiving key sexual health services. Ultimately, the long-term outcomes of a referral system are decreased STD, HIV, and pregnancy rates among adolescents and increased educational attainment. The framework's core components do not have to be addressed in any particular order and can be worked on simultaneously or one at a time. Each core component includes a set of recommended activities and tools that LEA can implement, use, and adapt. Throughout the project, CAI provided technical assistance to LEA on implementing the recommended activities and adaptation of tools; however, technical assistance is not required to implement the Core Components of a Referral System.

In the final year of implementation (2017), CAI conducted a case study among two LEA, consisting of in-depth interviews, to investigate contextual factors that could serve as facilitators or barriers to implement a system that connects students to sexual health services in two LEA.

\section{Methods \\ Study design}

Data was collected from January to April 2017 using a multiple case study design in two large urban school districts via in-depth qualitative interviews. The authors selected a case study approach, as it allowed for an "in-depth, multi-faceted understanding of a complex issue in its real-life context" [17].

\section{Application of the consolidated framework for} implementation research for interviews

We employed a well-known implementation science framework, the Consolidated Framework for Implementation Research (CFIR) for the case study, as it is essential to consider contextual factors at multiple levels in order for successful integration [18]. The CFIR consists of 39 constructs organized into five domains, that have been used in numerous studies to evaluate and identify key implementation facilitators and barriers [19-21]. 
The CFIR elucidates important contextual factors necessary for effective implementation of evidence-based practice and policies in real-world settings across five broad areas: intervention characteristics, outer setting, inner setting, characteristics of individuals, and process of implementation (see Table 1).

According to Damschroder et al, researchers may select CFIR constructs that are most relevant to the study setting to investigate, which has been applied successfully in previous studies [22-24]. Prior to developing the interview guides, the research team selected 20 constructs that were most relevant to the study setting and goals (see Additional file 1). CAI developed interview guides with approximately 25 open-ended questions guided and organized by the five CFIR domains and the 20 relevant constructs (see Additional file 2). The Core Components of a Referral System framework was used in the interview guides to ensure standardized definitions and terms describing a referral system (e.g., referral staff) were used. The interview guide was adapted for different groups of interviewees to adjust for relevant differences in roles or tasks.

Two CAI research staff conducted the interviews, each spanning 45-60 min. One interview was conducted via phone while the rest were conducted onsite. CAI digitally recorded and transcribed all interviews and kept files and data in a secure and confidential server during the study period. The interview data was coded using framework method for analysis which was based on all CFIR constructs and domains. The study protocol was reviewed and approved by Western Institutional Review Board and was determined to be exempt.

\section{Sample}

\section{Site selection}

CAI used convenience sampling to select two large urban school districts located in the Southeastern and Western U.S. for participation. These two LEA reflected geographic diversity, had different operations and policies, and during our tenure working with them as TA specialists, demonstrated varied approaches to implementation that we felt could best inform other LEA throughout the country. Further, the two LEA selected had strong relationships with our technical assistance providers, which ensured they would provide open and honest dialogues about successes and challenges in their work.

\section{Recruitment of participants}

CAI first contacted each of the LEA's program coordinators in November 2016 to request their participation in the study. After they agreed to participate, CAI used a snowball sampling technique to select interviewees by asking the program coordinator to identify diverse staff and stakeholders involved with planning, implementing, and evaluating their referral systems, making referrals at their priority schools, or providing referred students with sexual health services. This method of sampling was used since program coordinators were most knowledgeable of who the key stakeholders in implementing the referral system were.

\section{Participants}

A total of 19 staff members from both districts were interviewed from January to April 2017: 9 staff from the Southeast Site and 10 staff from the Western Site. Interviewees were classified as district staff (individuals who worked in the district offices, such as program directors and managers), school-building referral staff (individuals who worked directly in schools, such as teachers and school nurses), and community-based healthcare providers (CBHP) (individuals who worked at communitybased organizations as health educators or as testing coordinators, for example). See Table 2 for types of interviewees interviewed at each site.

\section{Analysis Interviews}

The "framework method", integrating both deductive and inductive approaches, guided the qualitative data coding and analysis [25]. All 39 CFIR constructs were used as codes to sort and organize qualitative data as interviewees provided valuable data related to constructs in addition to the pre-selected 20 constructs. Two independent coders coded the data using ATLAS.ti and met to compare and reconcile coding. After coding, common themes by construct and domain were put into a matrix and salient themes were identified for each site as well as across both sites.

Table 1 Application of CFIR Domains

\begin{tabular}{|c|c|}
\hline CFIR Domain & Description and application to current study \\
\hline $\begin{array}{l}\text { 1. Intervention } \\
\text { Characteristics }\end{array}$ & $\begin{array}{l}\text { Includes characteristics of the referral system and their potential influence on implementation. Examples of constructs } \\
\text { selected are: strength of evidence supporting the benefits of implementing an referral system in schools and perceived } \\
\text { difficulty implementing referral system (complexity). }\end{array}$ \\
\hline 2. Outer Setting & Describes the school districts' external political and social context through which implementation occurs. \\
\hline 3. Inner Setting & Includes the structural, political, and cultural context internally within the school district and school buildings. \\
\hline $\begin{array}{l}\text { 4. Characteristics of } \\
\text { Individuals }\end{array}$ & Describes those who are involved in the referral system, such as school-building staff. \\
\hline $\begin{array}{l}\text { 5. Process of } \\
\text { Implementation }\end{array}$ & Describes the referral system in its entirety from the planning stage, engaging staff and students, and evaluation. \\
\hline
\end{tabular}


Table $\mathbf{2}$ Interviewees by role and site

\begin{tabular}{|c|c|c|}
\hline & $\begin{array}{l}\text { Southeast Site } \\
(\boldsymbol{N}=9)\end{array}$ & $\begin{array}{l}\text { Western Site } \\
(\boldsymbol{N}=10)\end{array}$ \\
\hline $\begin{array}{l}\text { District-level staff } \\
\text { (e.g., program coordinators, evaluators, nursing program manager, school psychologist) }\end{array}$ & $3(33 \%)$ & $3(30 \%)$ \\
\hline $\begin{array}{l}\text { School-building referral staff } \\
\text { (e.g., teachers, nurses, counselors, student coordinators) }\end{array}$ & $3(33 \%)$ & $3(30 \%)$ \\
\hline $\begin{array}{l}\text { Community-Based Healthcare Provider } \\
\text { (e.g., executive director, clinical services director, health educators, HIV/AIDS program coordinators, education } \\
\text { and adolescent health manager) }\end{array}$ & $3(33 \%)$ & $4(40 \%)$ \\
\hline Total & 9 & 10 \\
\hline
\end{tabular}

\section{Results}

The following findings from the interviews at the two school districts are organized by the CFIR domains and summarized across the two sites and the various staff interviewed. CAI included quotes that demonstrate salient findings, labeled by the interviewees and their role and a randomly-generated identification number. Table 3 provides an overall summary of themes and quotes organized by CFIR domains and constructs.

\section{CFIR domain 1: intervention characteristics}

The CFIR domain, intervention characteristics, contains constructs that influence the implementation of the referral system (intervention), including evidence strength and quality, and complexity.

\section{Evidence strength and quality}

This construct investigated the extent to which interviewees felt that a school-based referral system will lead to the expected long-term outcomes-decrease in STI, HIV, and unintended pregnancy and increase in education attainment. Overall, interviewees reported that schools are in fact, an ideal location to implement referral system because students may not be able to access sexual health services at home or in their greater community. As one clinical services staff at a CBHP mentioned, "some young people don't have supportive parents, they live in a strict environment...for them it provides an opportunity to be able to get access to sexual health which is so important" (Interviewee 02-CBHP). In addition, most interviewees felt that a strong referral system can ease students' stress and anxiety in determining who and where to ask for help, thereby improving students' ability to focus at school, school attendance, and thus, educational attainment.

\section{Complexity}

During the interviews, participants overwhelmingly reported that the many layers of bureaucracy and the chaotic nature of the school system make implementation difficult. As one district staff mentioned, "the challenge we face is our bureaucracy...there's so many people that have to be involved that sometimes it hinders the process." (Interviewee 04-District
Staff). Another interviewee cited how "[The school district] is such a huge system that is very cumbersome and kind of in a transition mode. So there's a fair amount of chaos" (Interviewee 03-CBHP).

\section{CFIR domain 2: outer setting}

The second domain, Outer Setting, explores external factors that may influence the referral system, such as student needs, state and district policies, and partnerships.

\section{Needs and resources}

Most of the interviewees believed that connecting students to sexual health services is a high need. As one interviewee states, "On a scale of one to ten based on how much students need [sexual health services], I'd probably give it an 11". (Interviewee 02 -CBHP).

One reason is the high STI rates among the adolescents, specifically where the school districts are located: "We have a hot spot for chlamydia that we've seen, more outbreaks of syphilis than we have seen in the past." (Interviewee 02-CBHP). Interviewees also expressed that many students are sexually-active but are misinformed about risks, as they often share incorrect information with each other. Many interviewees expressed specific concerns for disproportionately affected populations, such as students of color and LGBTQ students.

\section{Cosmopolitanism}

In this context, cosmopolitanism can be defined as the degree to which each LEA is networked with other external organizations [17]. While each site has myriad local and state partnerships, two types of organizations emerged as crucial partners: local health departments and CBHP. Interviewees noted that through the planning and implementation of the referral system, partnerships among these organizations were strengthened. "It was a tenuous relationship in the past but...this grant has really increased our ability to access the school board and have a seat at the table" (Interviewee 03-CBHP).

Specifically for local health departments, the interviewees expressed that they played a prominent role in supporting professional development sessions for teachers and staff, 
Table 3 Summary of emergent themes from qualitative interviews that influence referral system implementation

\begin{tabular}{|c|c|}
\hline CFIR Construct & Themes \\
\hline \multicolumn{2}{|c|}{ I. Intervention Characteristics } \\
\hline $\begin{array}{l}\text { Evidence Strength and } \\
\text { Quality }\end{array}$ & $\begin{array}{l}\text {-Schools are an ideal location to address the epidemic of } \\
\text { STIs, HIV, and unintended pregnancy }\end{array}$ \\
\hline
\end{tabular}
Quotations

Quality STIS, HIV, and unintended pregnancy

-The referral system can help students achieve better academic outcomes

Complexity

\section{Outer Domain}

Needs and Resources

Cosmopolitanism

State and District Policies

\section{Inner Setting}

Networks and Communications

Organizational Culture and Access to Knowledge and Information

Leadership Engagement Principals have a lot of authority on the day-to-day operation of schools but their acceptance and commitment to implementing a sexual health referral system varies

\footnotetext{
- District-level leadership (e.g., superintendent) and community-based health service providers were described as supportive, involved, and motivated
}

Available Resources

-A full-time referral staff as crucial in the success of the referral system

-SBHC were identified as important resources

\section{Characteristics of Individuals}

Knowledge and Belief

-Staff who were actively involved were most knowledgeable about the logistics of referral-making and the policies. School staff and students have a general knowledge of the staff to refer to for health-related questions.
"Schools represent... a really important social institution in the lives of young people and means by which we can get young people aware about resources in the community, I think that they're a key player." (Interviewee 07-CBHP)

When students can access SHS referrals from school staff, they "have the ability to focus more on what they are here to do every day, which is to get their education." (Interviewee 16-District Staff

"It's such a huge system that is very cumbersome and kind of in a transition mode. So there's a fair amount of chaos, in my experience, in just dealing with the system" (Interviewee 3 - (BHP)

"They've all got experience, they've all done everything, if you listen to their stories. But they don't even know basics about female and.... male anatomy." Interviewee 14 - School Building Referral Staff)

"We have a fantastic relationship with the health department... We work hand-in-hand." (Interviewee 18 District Staff)

"I think sometimes policy creates, or has the potential to create, barriers because people don't understand realistically what the policy is saying." (Interviewee 16 -District Staff)

The nursing staff "are broken into clusters...I think I have more actual discussion of how to handle [SHS referrals] at our cluster level than I do at the district level, just because those are the nurses I see more often, and we have the same student populations." (Interviewee 15 - School Building Referral Staff)

"It's such a culturally conservative environment that most people who work with teenagers and young people are really afraid of sexuality, and they're afraid to really go there. (nterviewee 3 - CBHP)

"The principals still almost have reign over their school... And the principals can decide the way that things run in their school. And they could be, for instance, homophobic or even sex negative versus sex positive." (Interviewee 4 - District Staff)

"The leaders here are really on board with [the referral system], and they really encourage us to do a lot more and get the program really out there to do a lot for the students. They're very supportive" (Interviewee 8 - School Building Referral Staff)

"Knowing that [the referral staff] is there five days a week and knowing the level of conversations that she has with kids... and the time that she spends linking and connecting families to services, I know why kids are doing better in her school." (Interviewee 16 - District Staff)

"...Our school staff are pretty well-informed about the services that the $[S B H C]$ offers. They may not know how the logistics works off campus... And I don't think they really know all of the law surrounding [state's family planning law]." (Interviewee 19-CBHP)

\section{Process}


Table 3 Summary of emergent themes from qualitative interviews that influence referral system implementation (Continued)

\begin{tabular}{cll}
\hline CFIR Construct & Themes & Quotations \\
\hline Engaging & -The role of formally appointed implementation leader was & "Well, without somebody advocating for [the SHS referral \\
& essential in supporting implementation, especially in \\
& engaging key stakeholders and provision of professional \\
development & $\begin{array}{l}\text { happened. So I give [Program Coordinator] a lot of credit." } \\
\text { (Interviewee 13-CBHP) }\end{array}$ \\
Reflecting and & $\begin{array}{l}\text {-Tracking referrals was identified as challenging as current } \\
\text { Evaluating }\end{array}$ & "So I think probably... some of the referrals aren't being \\
& efforts that don't necessarily result in a referral & $\begin{array}{l}\text { tracked the way that they should be. But we don't really have } \\
\text { systems in place that would support that process" } \\
\text { (Interviewee 2-CBHP) }\end{array}$ \\
\hline
\end{tabular}

offering sexual health services to students through SBHC and assisting LEAs with monitoring and evaluation activities.

CBHP, described as "youth-friendly, LGBT-inclusive, more up-to-date than typical clinicians on the latest birth control methods and STI testing, HIV testing" (Interviewee 11-District Staff), also play active and crucial roles in the implementation of the referral system. Interviewees reported their involvement in providing sexual health services to students, delivering training and technical assistance to other CBHP, and in creating resources for LEAs (e.g., referral guides, staff professional development curricula). Interviewees also felt that these partnerships increased CBHP's awareness of state and local policies regarding minors' access to health services.

Additionally, interviewees from the Southeastern site mentioned that the relationship between the CBHP and the LEA has strengthened significantly in the last several years. In the past, the CBHP was not allowed on school campuses due to the larger political climate and a restrictive district policy. Several interviewees mentioned that through collaborating to create a referral system, the partnership has been strengthened: "people in the school system are much more open now and understand that having a relationship with a youth advocacy organization is a strengthening component". (Interviewee 03-CBHP).

\section{State and district policies}

While interviewees identified how some state laws facilitate referral system-the Western site's state law allows students to access STI services (including HIV testing and treatment) without parental consent, other policies serve as barriers-the Southeastern state's guidance for abstinence-only education is an obstacle for schools to deliver comprehensive sexual health education, as well as the law that contraceptive counseling cannot be provided on school campus: "not being able to talk about condoms or any contraceptives in the school is a humongous barrier" (Interviewee 12-CBHP). Further, the conflict between state- and district-level policies was observed to be a barrier in the Western site; while the state law allows for student access to sexual health services without parental consent, district policy is more restrictive: "Sexual health services has to be [provided] through a SBHC with a contract with the district. ... There are very few [SBHC] in our school district, and each of those has their own contract about what can or can't be given" (Interviewee 18-District Staff).

\section{CFIR domain 3: inner setting}

The third domain, Inner Setting, refers to the location, structural, social, organization, and cultural characteristics of where the intervention takes place. Constructs that were explored included networks and communications, culture and climate, leadership, and available resources.

\section{Networks and communications}

Networks and communications refer to the nature and quality of social networks and of formal and informal communications within the school district. Each site had distinct networks and communication systems, drawing from strong and pre-existing staffing structures and innovative marketing and communication approaches, to facilitate referral system implementation. In the Western site, the referral system was supported by nurses across different schools organized into teams called "clusters." These clusters met monthly and often discussed successes and challenges related to referring students to sexual health services.

In the Southeastern site, the referral system was supported by a palm-sized 'chat card,' a communication tool used by staff to refer students to services. Chat cards have information about teen health center locations, types of services offered, and contact information. However, many interviewees from this site noted that the 'chat cards' might not be directive enough to link students to sexual health services.

\section{Organizational culture and access to knowledge and information}

In the Western site, most staff mentioned that the organizational culture is supportive of sexual health prevention services. However, they also mentioned that the county is more conservative, relative to the rest of the state, and that negative attitudes and stigma towards sexual health education and services exist. Interviewees felt that other school staff did not feel capable of promoting the referral system (e.g., linking students to designated referral staff or giving referral guides to students) due to general fears 
related to parents, legal issues, and general lack of knowledge about the referral system. One interviewee expressed that, "there is this nervousness that principals and other people have when kids go to confidential appointments when they're entrusted by their parents to be at school" (Interviewee 16-District Staff). Similarly, many staff in the Southeastern site highlighted the culturally conservative climate as negatively impacting the referral system.

\section{Leadership engagement}

Since school principals have a lot of authority on the dayto-day operation of schools, they can influence the degree of referral system implementation. Most interviewees at both sites reported that principals' acceptance and commitment to implementing a sexual health referral system varies. A few of the interviewees stated that schoolbuilding level champions were at times hard to identify due to lack of buy-in from principals. However, districtlevel leadership (e.g., superintendents) and CBHP were described overall as supportive, involved, and motivated. As one interviewee stated, "the leaders here are on board with it, and they really encourage us to get the program out there for the students". (Interviewee 07-CBHP).

\section{Available resources}

Many interviewees discussed the importance of having a full-time referral staff as crucial in the success of the referral system. Further, the Southeastern site mentioned that because the district is spread out geographically and that public transportation is limited, their five SBHC are critical resources for students. As one interviewee stated, "it's hard for a lot of the students to get to health clinics unfortunately, which is why it's important that we are in the schools." (Interviewee 05-District Staff). Challenges were also mentioned around SBHC, which included space, staffing, inadequate hours, and difficulty for students who attend other schools to access.

\section{CFIR domain 4: characteristics of individuals}

The fourth domain describes the individuals involved with the referral system, such as district staff, school staff, CBHP and leadership. Specifically, these individuals' knowledge and beliefs regarding sexual health services emerged as a salient construct.

\section{Knowledge and belief}

Across the two sites, interviewees reported that staff who were the most actively involved in referral system (e.g., referral staff, program coordinator, champions) were the most knowledgeable, especially regarding the logistics of making a referral, policies, and promoting student access to health services. Interviewees stated that knowledge about the referral system is generally higher among all staff and students if the school has a SBHC onsite or if the referral system is heavily marketed through posters, flyers, and/or announcements.

However, interviewees reported that beliefs towards the referral system among school-building staff were mixed. Negative attitudes and the stigma of discussing sexual health came up as major barriers across the two sites. In the Southeastern site, interviewees reported that their colleagues are hesitant to make referrals as they do not want to appear that they are endorsing adolescents having sex. One district staff said: “...We're in the Bible Belt and I think some of the mindsets... about the way that children should be behaving... hinder the referral process." (Interviewee 04-District Staff). Similarly, in the Western site, one interviewee stated "Some people are worried about kids leaving campus and they don't agree with the law and they question the law" (Interviewee 16-District Staff).

Additionally, many interviewees stated that despite having supporting policies in place at the state- or districtlevel, lack of knowledge or misunderstanding of the policies are barriers to sexual health service provision. Laws surrounding consent are often difficult for school staff to comprehend because it is outside of typical practice for schools to allow students to do anything without parental consent: "The school system does not do anything with young people without parental consent and engagement, except for in rare situations" (Interviewee 03-CBHP). Furthermore, many interviewees mentioned that sometimes, local- and state-policies conflict, which is confusing for school and district staff.

\section{CFIR domain 5: process of implementation}

The last domain looks at the entire process of the referral system through different stages of implementation. Salient constructs in this domain include engaging staff and students in the referral process and reflecting and evaluating.

\section{Engaging}

Many interviewees spoke of the necessity of the Program Coordinator position to employ different strategies to engage the appropriate individuals to ensure successful implementation and use of the referral system. Examples of these strategies include: recruiting and training referral staff on referral procedures and minor consent and confidentiality laws; meeting with district and school leaders to provide updates on referral system implementation; establishing and maintaining formal partnerships with CBHPs; and distributing communication and marketing materials in schools. As one CBHP manager stated, "I've been really impressed with her ability to sort of navigate all of this... a lot of it just falls on her. She's really coordinating with everyone and working with the schools and coordinating with the partner agencies." 
Specifically, professional development proved to be important to engage designated referral staff (e.g., counselors, school nurses), to ensure that they know about Core Components of a Referral System and the health resources available for students, and to increase their knowledge in local STI, HIV, and pregnancy statistics.

Engagement of students mostly occurred through posters, flyers, palm cards, school announcements, banners, and calendars. Social media approaches (e.g., Instagram, Snapchat) are currently being developed as well in these sites.

\section{Reflecting and evaluating}

Methods to conduct ongoing tracking and evaluating of the referral system varied depending on the school district. In the Southeastern site, the local health department develops tracking tools, collates data, and ensures data quality. In the Western site, the program coordinator developed a paper log for nurses to track data and distribute a monthly survey to collect data. Across the two sites, tracking referrals was identified as very difficult as the current data collection systems does not capture passive referrals (e.g., self-referral when student seeks out sexual health services on their own after seeing a poster) or efforts that do not result in a referral. Further, only the staff who were most involved in referral system implementation regularly receive data reports and take the time to use data to evaluate their work.

\section{Discussion}

Overall, this study demonstrates, taking into account inner and outer contexts, that schools can be ideal locations to address STI, HIV, and unintended pregnancy prevention among adolescents. Our findings also indicate that referral systems linking students to sexual health services are capable of being integrated and implemented across different kinds of settings; the Southeastern site implemented their referral system in a less favorable implementation climate (e.g., more restrictive state policies, fewer resources to leverage, etc.), whereas the Western site had a more supportive political climate. The use of an implementation science approach allowed us to identify, explore, and report on factors across multiple domains that influenced implementation at each site, offering a useful framework for other LEA to consider when beginning or continuing to refine a referral system in their districts, regardless of differing policies and/or environments.

A salient facilitator identified within the outer setting is the extent of collaboration between schools and external partners, such as community-based healthcare providers. True collaboration, when there was the highest level of partnership, consisted of multiple factors: a shared vision/ outcome, shared ownership of the system by health and education partners, and formalized agreements [26]. The study demonstrates how strong partnerships often took the burden off of school staff to be the only ones to refer students to sexual health services, especially if they lacked confidence or capacity to do so. By collaborating and referring out to other organizations, LEA were able to share responsibility and also ensure provision of culturallycompetent services. Therefore, inviting community agencies and organizations with similar visions of improving student health and academic outcomes to partner with local school districts is another promising approach for strengthening school-based referral systems [27].

Other influencing factors with the outer context domain included statewide policies, which were shown to heavily impact referral system implementation in both settings. Not only did prohibitive policies emerge as a significant barrier, we also found that having supportive statewide policies alone are not always sufficient. Interviewees described how important it is for implementers to be knowledgeable of their districts' policies in order to make progress in their referral systems.

The importance of being knowledgeable about policies also emerged during questions covering the inner setting and characteristics of individuals domains. Lack of knowledge regarding policies led to school staff's avoidance of implementing aspects of the referral system (e.g., not providing referrals or referral guides to students) for fear of being noncompliant with policies. As we saw in the Western site, even in a supportive sexual health environment, school staff who serve as gatekeepers (i.e., principals, office staff, hall monitors) were unaware of the policies of student access to services; this gap in awareness and knowledge has been corroborated in past studies as barriers to sexual health and nutrition education and policy implementation within schools [28-31]. Equally important to increasing staffs knowledge of stateand district-level sexual health policies is addressing negative attitudes and stigma. Interviewees described how the presence of negative or unsupportive attitudes regarding the referral system, due to societal stigma surrounding teens accessing sexuality education and health services, was a significant barrier to implementation. Acknowledging and addressing stigma and conservative views around sexual health, especially in the beginning phases of implementation, is critical [32,33]. One way to mitigate these gaps in knowledge and negative attitudes may be to increase guidance and education regarding state and local policies via professional development and trainings in schools.

Facilitators identified within the inner setting domain related to identification and leveraging of existing resources, communication networks, and workflows to ensure effective implementation. For example, the Western site employed nurses as referral staff and leveraged the nurse clusters' well-established shared vision of improving student wellness, formal communication structure, peer collaboration, and feedback and review among 
peers. Understanding different workflows and where there is a potential for integration involves assessment of school district's existing networks, systems, and partnerships to the wider community, which could then promote implementation success.

A key barrier identified within the inner context was school district bureaucracy. Previous studies have also identified how bureaucratic factors, such as lack of supported staff time to support implementation and/or lack of funding and prioritizing by school administration, are barriers to implementing new school-based policies and health promotion programs [34, 35]. As demonstrated in the case studies, the role of the program coordinator provided crucial oversight and structure for the program, and without funding for this full-time dedicated coordinator position, the referral systems may not have been successfully implemented. Therefore, given that funding is often challenging to secure and hiring new staff is not always feasible, it is recommended that LEA take advantage of already formalized organizational structures, staffing, and workflows where possible.

\section{Limitations}

This study has several limitations to report. First, since we selected only two LEA for inclusion in these case studies, findings may not be generalizable to all LEA throughout the U.S. Also, the interviewees were selected via snowball sampling methods, and therefore, sampling bias may be an issue as the program coordinators may have nominated interviewees who they know well and with whom they have similar traits and characteristics. Another limitation was that the interview guide was developed using 20 selected CFIR constructs, without assessing for all of the CFIR constructs, which could have limited our findings. However, we found that interviewees still provided data related to constructs in addition to the pre-selected 20. Lastly, the perceptions of students, who were the beneficiaries of the referral system, were not explored. Thus, there are likely additional important experiences and perceptions of the referral system not captured by the current study.

\section{Conclusions}

The study's use of the CFIR offered a helpful framework to investigate two distinct school-based referral systems, enabling us to identify and describe the complexity of barriers and facilitators involved in establishing a schoolbased referral system. Three general recommendations emerged from the findings which may have applicability for other LEA interested in implementing a referral system. First, LEA would benefit from appointing key stakeholders who are supportive and will lead referral system implementation; these stakeholders include: school leaders (e.g., principles, superintendents) and champions.
School leaders can facilitate a culture shift within a school community and champions are considered imperative to get the project up and running and to integrate intervention practices into current school workflows [36]. Second, LEA should evaluate readiness prior to implementing a referral system and identify contextual factors that will serve as barriers or facilitators to implementation. For example, surveys can be distributed to school staff to assess knowledge, skills, self-efficacy, and attitudes towards providing SHS referrals to students and assessments can be performed to evaluate existing resources that can be leveraged. Third and lastly, based on the results the readiness assessment, LEA should identify strategies to address barriers and create action plans to prioritize goals, create actionable steps towards goals, and delineate roles and responsibilities [37, 38]. For example, a professional development plan for school staff may be warranted to increase knowledge of minor consent laws and policies and decrease negative attitudes and stigma towards sexual health services. Applying these recommendations as well as deliberately assessing presence of facilitators and barriers prior to and during implementation, especially in a complicated school environment, is essential to ensuring adequate preparation and readiness and ultimately improving delivery of vital services to students.

\section{Supplementary information}

Supplementary information accompanies this paper at https://doi.org/10. 1186/s12913-020-05147-z.

Additional file 1. Consolidated Framework for Implementation Research (CFIR) Domains and Constructs. The table depicts all 39 constructs organized by the 5 CFIR domains as originally published by Damschroder et al. The CFIR constructs with * were selected for the development of the interview guides for this case study. All 39 constructs were used as codes in the analysis phase.

Additional file 2. Interview Guide. The interview guide, organized by CFIR domain and interviewee role, was used to conduct the interviews with three types of interviewees, district-level staff, school-building referral staff, and community-based healthcare providers.

\section{Abbreviations}

CAl: Cicatelli Associates Inc.; CDC: Centers for Disease Control and Prevention; CBHP: Community-based healthcare provider; CFIR: Consolidated Framework for Implementation Science; HIV: Human immunodeficiency virus; LEA: Local education agency; SBHC: School-based health center; STI: Sexually transmitted infections; U.S.: United States

\section{Acknowledgements}

The authors thank Sandra Leonard and Malaika Washington (Centers for Disease Control and Prevention) for their support in the initial design of the study. We also thank the program coordinators at each of the study sites for their assistance in supporting the study and recruitment of participants.

\section{Authors' contributions}

EL and KW took the lead in conceptualizing and writing the manuscript, collecting data, and analyzing the data. EL and AB designed the interview guides, with input provided by LS and DM. AB, LS, and DM provided ongoing feedback and critique of the findings and conceptualizing of the manuscript. EL, LS, and KW revised the manuscript. All authors read and approved the final manuscript. 


\section{Funding}

This work was supported by the Cooperative Agreement \#1U87PS004164-03 from the Centers for Disease Control and Prevention. Its contents are solely the responsibility of the authors and do not necessarily represent the official views of the Centers for Disease Control and Prevention. The funding body had a role in the initial conceptualization of the study but had no role in the design of the study, the collection, analysis, and interpretation of data and in writing the manuscript.

\section{Availability of data and materials}

The qualitative data used and analyzed for the current study are not publicly available as individual privacy may be compromised. Requests from data may be available upon reasonable request from the corresponding author.

\section{Ethics approval and consent to participate}

The study protocol, including the verbal consent process, was reviewed and approved by Western Institutional Review Board and was determined to be exempt research on January 11, 2017 (WIRB Work Order \# 1-988019-1). Prior to data collection, verbal consent was provided by all participants to participate in the study, be audio-recorded, and have their quotes published on the basis of anonymity. Participants were provided with a document with information about the study. Verbal consent was determined the best method for obtaining consent from the interviewees as it was least burdensome and was most natural during the process of beginning the qualitative interviews. Our verbal consent process incorporated the three key features of information consent: 1) disclosing to interviewees information needed for them to make an informed decision, 2) ensuring that they understand the information disclosed, and 3) ensuring that participants know that participation in the study is voluntary.

\section{Consent for publication}

Not applicable.

\section{Competing interests}

The authors declare that they have no competing interests.

\section{Author details}

${ }^{1}$ Cicatelli Associates, Inc. (CAI), 505 Eighth Ave. Suite 1900, New York, NY 10018, USA. ${ }^{2}$ Franklin and Marshall College, College Square 931 Harrisburg Ave. 1st Floor, Lancaster, PA 17603, USA.

Received: 21 June 2019 Accepted: 24 March 2020

Published online: 07 April 2020

\section{References}

1. Centers for Disease Control. STDs in Adolescents and Young Adults. Atlanta; 2018. Available from: https://www.cdc.gov/std/stats17/adolescents.htm. Cited 2019 April 30.

2. Centers for Disease Control. Press Release: New CDC analysis shows steep and sustained increases in STDs in recent years. Atlanta; 2018. Available from: https://www.cdc.gov/nchhstp/newsroom/2018/2018-std-preventionconference.html. Cited 2019 April 30.

3. Finer LB. Unintended pregnancy among U.S. adolescents: accounting for sexual activity. J Adolesc Health. 2010;47(3):312-4. https://doi.org/10.1016/j. jadohealth.2010.02.002

4. Forhan SE, Gottlieb SL, Sternberg MR, Xu F, Datta SD, McQuillan GM, et al. Prevalence of sexually transmitted infections among female adolescents aged 14 to 19 in the United States. Pediatrics. 2009;124(6):1505-12.

5. Finer $\mathrm{L}$, Zolna M. Declines in unintended pregnancy in the United States, 2008-2011, 2016. N Engl J Med. 2016;374:843-52. https://doi.org/10.1056/ NEJMsa1506575 Accessed 31 Jan 2020.

6. Kann L, McManus T, et al. Youth Risk Behavior Surveillance-United States, 2017. Morb Mortal Wkly Rep (MWWR). 2018; https://www.cdc.gov/ healthyyouth/data/yrbs/pdf/2017/ss6708.pdf. Accessed 30 Apr 2019.

7. National Center for Education Statistics. Back to school statistics. 2011. https://nces.ed.gov/fastfacts/display.asp?id=372. Accessed 30 Apr 2019.

8. U.S. Department of Health and Human Services Office of Disease Prevention and Health Promotion. 2017. https://www.healthypeople.gov/2020/topicsobjectives/topic/Access-to-Health-Services. Accessed 30 Apr 2019.
9. Arenson M, Hudson PJ, Lee N, Lai B. The Evidence on School-Based Health Centers: A Review. Glob Pediatr Health. 2019;6:2333794X19828745. https:// doi.org/10.1177/2333794X19828745

10. Fisher R, Danza P, McCarthy J, Tiezzi L. Provision of contraction in New York City School-based health centers: impact on teenage pregnancy and avoided costs, 2008-2017. Perspect Sex Reprod Health. 2019;51(4):201-9. https://doi.org/10.1363/psrh.12126 Accessed 4 Feb 2020.

11. Bersamin M, Paschall MJ, Fisher DA. Oregon School-Based Health Centers and Sexual and Contraceptive Behaviors Among Adolescents. J Sch Nurs. 2018;34(5):359-66. https://doi.org/10.1177/1059840517703161 Accessed 4 February 2020.

12. School-Based Health Alliance. 2013-2014 Digital Census Report. http:// censusreport.sbh4all.org/. Accessed 30 April 2019.

13. Boonstra HD. Meeting the Sexual and Reproductive Health Needs of Adolescents in Schools. 2015 https://www.guttmacher.org/gpr/2015/04/ meeting-sexual-and-reproductive-health-needs-adolescents-school-basedhealth-centers. Accessed 30 Apr 2019.

14. Cicatelli Associates Inc. (CAI), and National Coalition of STD Directors (NCSD), and Centers for Disease Control (CDC). Developing a Referral System for Sexual Health Services: An Implementation Kit for Education Agencies. 2015. http://www.connectionsforstudentsuccess.org/index.php/ referral-kit-download/referral-kit-download-2. Accessed 30 Apr 2019.

15. Dittus PJ, De Rosa CJ, Jeffries RA, Afifi AA, Cumberland WG, Chung EQ, Martinez E, Ethier KA. The project connect health systems intervention: Linking sexually experienced youth to sexual and reproductive health care J Adolesc Health. 2014;55:528-34. https://doi.org/10.1016/j.jadohealth.2014. 04.005 Accessed 30 Apr 2019.

16. Rasberry CN, Liddon N, Adkins SH, Lesesne CA, Hebert A, Kroupa E, Rose ID, Morris E. The importance of school staff referrals and follow-up in connecting high school students to HIV and STD testing. J Sch Nurs. 2017;33(2):143-53. https://doi.org/10.1177/1059840516658695 Accessed 30 April 2019.

17. Crowe S, Cresswell K, Robertson A, Huby G, Avery A, Sheikh A. The case study approach. BMC Med Res Methodol. 2011;11:100. https://doi.org/10. 1186/1471-2288-11-100 Accessed 30 April 2019.

18. Atkins MS, Rusch D, Mehta TG, Lakind D. Future directions for dissemination and implementation science: Aligning ecological theory and public health to close the research to practice gap. J Clin Child Adolesc Psychol. 2016;45:21526. https://doi.org/10.1080/15374416.2015.1050724 Accessed 30 Apr 2019.

19. Kramer JB, Cote SD, Lee DL, Creekmur B, Saliba D. Barriers and facilitators to implementation of VA home-based primary care on American Indian reservations: a qualitative multi-case study. Implement Sci. 2017;12(1):109. https://doi.org/10.1186/s13012-017-0632-6 Assessed 30 Apr 2019.

20. Leeman J, Wiecha JL, Vu M, Blitstein JL, Allgood S, Lee S. School health implementation tools: a mixed methods evaluation of factors influencing their use. Implement Sci. 2018;13(1):48. https://doi.org/10.1186/s13012-0180738-5 Accessed 30 Apr 2019.

21. Selove R, Foster M, Mack R, Sanderson M, Hill PC. Using an Implementation Research Framework to Identify Potential Facilitators and Barriers of an Intervention to Increase HPV Vaccine Uptake.J Public Health Manag Pract. 2017;23(3):e1-9. https:/doi.org/10.1097/PHH.0000000000000367 Accessed 30 Apr 2019.

22. Damschroder LJ, Aron DC, Keith RE, Kirsh SR, Alexander JA, Lowery JC. Fostering implementation of health services research findings into practice: a consolidated framework for advancing implementation science. Implement Sci. 2009;4:50. https://doi.org/10.1186/1748-5908-4-50 Accessed 22 May 2019.

23. Paulsen MM, Varsi C, Paur I, Tangvik RJ, Andersen LF. Barriers and facilitators for implementing a decision support system to prevent and treat diseaserelated malnutrition in a hospital setting: qualitative study. JMIR Form Res. 2019;3(2):e11890. https://doi.org/10.2196/11890 Accessed 10 Feb 2020.

24. Soi C, Gimbel S, Chilundo B, et al. Human papillomavirus vaccine delivery in Mozambique: identification of implementation performance drivers using the Consolidated Framework for Implementation Research (CFIR). Implement Sci. 2018;13:151. https://doi.org/10.1186/s13012-018-0846-2 Accessed 4 Feb 2020.

25. Gale NK, Heath G, Cameron E, et al. Using the framework method for the analysis of qualitative data in multi-disciplinary health research. BMC Med Res Methodol. 2013;13:117. https://doi.org/10.1186/1471-2288-13-117 Accessed 7 Feb 2020

26. The National Network for Collaborative. Collaboration FrameworkAddressing Community Capacity. http://www.uvm.edu/crs/nnco/collab/ framework.html. Accessed 30 Apr 2019 
27. Rocha AC, Duarte C. Factors facilitating implementation of school-based sexuality education in Portugal. Eur I Contracept Reprod Health Care. 2006; 21(1). https://doi.org/10.3109/13625187.2015.1057809 Accessed 9 Feb 2020.

28. Dickson $E$, Parshall $M$, Brindis CD. Isolated voices: perspectives of teachers, school nurses, and administrators regarding implementation of sexual health education policy. J Sch Health. 2019;90(2):88-9. https://doi.org/10. 1111/josh.12853 Accessed 9 Feb 2020.

29. Reis J, Seidl A. School adminstrators, parents, and sex education: a resolvable paradox? Adolescence. 1989;24(95):639-45 Accessed 9 Feb 2020.

30. Lanier WA, Wagstaff RS, DeMill JH, Friedrichs MD, Metos J. Teacher awareness and implementation of food and physical activity policies in Utah elementary schools, 2010. Prev Chronic Dis. 2012;9:110091. https://doi. org/10.5888/pcd9.110091 Accessed 30 Apr 2019.

31. McCaughtry N, Martin JJ, Fahlman M, Shen B. Urban health educators' perspectives and practices regarding school nutrition education policies Health Educ Res. 2012;27(1):69-80. https://doi.org/10.1093/her/cyr101 Accessed 30 Apr 2019

32. Craig G, Stanley N. Visibility, Immobility and Stigma: Young People's Use of Sexual Health Services in Rural Areas. Child Soc. 2006;20(3). https://doi.org/ 10.1002/CHI.880 Accessed 30 Apr 2019.

33. Green A, Willging C, Ramos M, Shattuck D, Gunderson L. Factors Impacting Implementation of Evidence-Based Strategies to Create Safe and Supportive Schools for Sexual and Gender Minority Students. J Adolesc Health. 2018;63(5): 643-8. https://doi.org/10.1016/j.jadohealth.2018.06.004 Accessed 30 Apr 2019.

34. Langley AK, Nadeem E, Kataoka SH, Stein BD, Jaycox LH. Evidence-based mental health programs in schools: barriers and facilitators of successful implementation. Sch Ment Heal. 2010;2(3):105-13. https://doi.org/10.1007/ s12310-010-9038-1 Accessed 30 April 2019.

35. Hall WJ, Schneider M, Thompson D, Volpe SL, Steckler A, Hall JM, Fisher MR, et al. School factors as barriers to and facilitators of a preventive intervention for pediatric type 2 diabetes. Transl Behav Med. 2014;4(2):13140. https://doi.org/10.1007/s13142-013-0226-z Accessed 30 Apr 2019

36. Storey KE, Montemurro G, Flynn J, et al. Essential conditions for the implementation of comprehensive school health to achieve changes in school culture and improvements in health behaviours of students. BMC Public Health. 2016;16(1):1133. https://doi.org/10.1186/s12889-016-3787-1 Accessed 11 Feb 2020

37. Powell BJ, Waltz TJ, Chinman MJ, et al. A refined compilation of implementation strategies: results from the Expert Recommendations for Implementing Change (ERIC) project. Implement Sci. 2015, 10(21). https:// doi.org/10.1186/s13012-015-0209-1. Accessed 10 February 2020.

38. Moore JB, Carson RL, Webster CA, et al. The Application of an Implementation Science Framework to Comprehensive School Physical Activity Programs: Be a Champion! Front Public Health. 2018, 5:354. https:// doi.org/10.3389/fpubh.2017.00354. Accessed 10 February 2020.

\section{Publisher's Note}

Springer Nature remains neutral with regard to jurisdictional claims in published maps and institutional affiliations.

\section{Ready to submit your research? Choose BMC and benefit from:}

- fast, convenient online submission

- thorough peer review by experienced researchers in your field

- rapid publication on acceptance

- support for research data, including large and complex data types

- gold Open Access which fosters wider collaboration and increased citations

- maximum visibility for your research: over $100 \mathrm{M}$ website views per year

At BMC, research is always in progress.

Learn more biomedcentral.com/submissions 